\title{
Research on the Accurate Computer Music Composition Mode under the Background of Big Data
}

\author{
Sui Wu \\ Hunan City University, \\ Yiyang,Hunan,413000 China
}

\begin{abstract}
In this paper, we conduct research on the accurate computer music composition mode under the basic background of big data. From the perspective of music theory, in terms of micro notes jump within a certain range, will not cause discomfort, notes there are upward at the same time, the downward makes the whole piece will not boring. From the macroscopic, the nature of the chord with internal connection between motivation and motivation as support, at the same time, the whole range can maintain in a certain range, making sound effects is can let a person feel cheerful. Computer science first occupied is music in the music sound production and the propagation of position as it is mainly used for digital audio clips and automated synthesis. But there is a certain distance away from the traditional concept of composition. Therefore, our research proposes the innovative perspective to deal with the issues for achieving the better performance.
\end{abstract}

Keywords- Big Data, Composition Mode, Accurate, Computer Music, Theoretical Research.

\section{Introduction}

Called the contemporary digital age is not too much, it was useful in practice, the social developments of recent years has been proved that brought by the digital process in all aspects of the social change is fundamental. Digital can make many industries on a large scale of production and production, a large number of reduce the cost of production and threshold, in terms of the music, we have every reason to look for to give us life bring music characteristics of modern color science and technology, to look for it to human cherished value the benefits and threats. In fact in the digital environment, ecological and great changes have taken place in the music. Digital continuous variation of the sound signal can be converted to a string of general separation unit, rapid flow of digital information system exist in the relationship between numeric code, music creation has become a large number of the simulacra paste, core loop, sampling, as well as the synthetic process [1-3].

Digital technology will be to a full range of infiltration of music, the music creation system of each elements are present a diverse landscape, then there will be a new form of music, traditional music forms will be challenged, even in music education aspects. Digital technology to create the music works with the method of the deconstruction of the music art, so as to achieve a more free to note synthesis condition. Composer and arranger and meet the development of new modern opportunities. Emerging composers are with new ideas, new experience and feeling to answer the challenge of the modern science and technology, but also provides a wider space, singer to general modern audience expectations with the infinite development and openness of the Internet, which will contain the new opportunities for the development of the music art [4].

Score of the composition of the traditional way is to write on paper, composer with a hand-written way constantly change, a dozen or even dozens of music records and changes are very inconvenient, and difficult to instantly get sound effects. With the aid of computer aided composing, the composer can take advantage of the computer generated a lot of music in a very short time. Computer music analysis is based on some sort of the computer music description. Points from the core angle of music elements, it 
can be divided into rhythm, tones retrieval, tone recognition, style, divided into emotional extraction and so on the many kinds of the research direction as the follows. (1) Style and emotional extract: music style and music emotion is very abstract thing, often is not a specific standard. So dividing style and the emotion is more difficult. Through analyzing the music expression parameters automatically set up a system based on the instance, and then system through synthetic technology will get the expression of parameters used in the new tone. (2) Beat tracking, beat tracking model is almost as some early study of records such as music, melody, tone of the byproducts. Beat the concept of extended to include age and stage two aspects, this approach allows the tracker considering several possible state, similar to the history of the mechanism, but not just rely on the confidence measure, and adopted a method of reliability, this at every given time there will be a series of dynamic state performance and so far, the interpretation of the most trusted. (3) Tones to retrieve the scientists put forward four tones matching algorithm based on the MIDI, on the basis of comparison that proposes algorithm based on time frame to tone and the matching of time [5-6].

In this paper, we conduct research on the accurate computer music composition mode under the background of big data. The following figure shows the sample computer music platform.

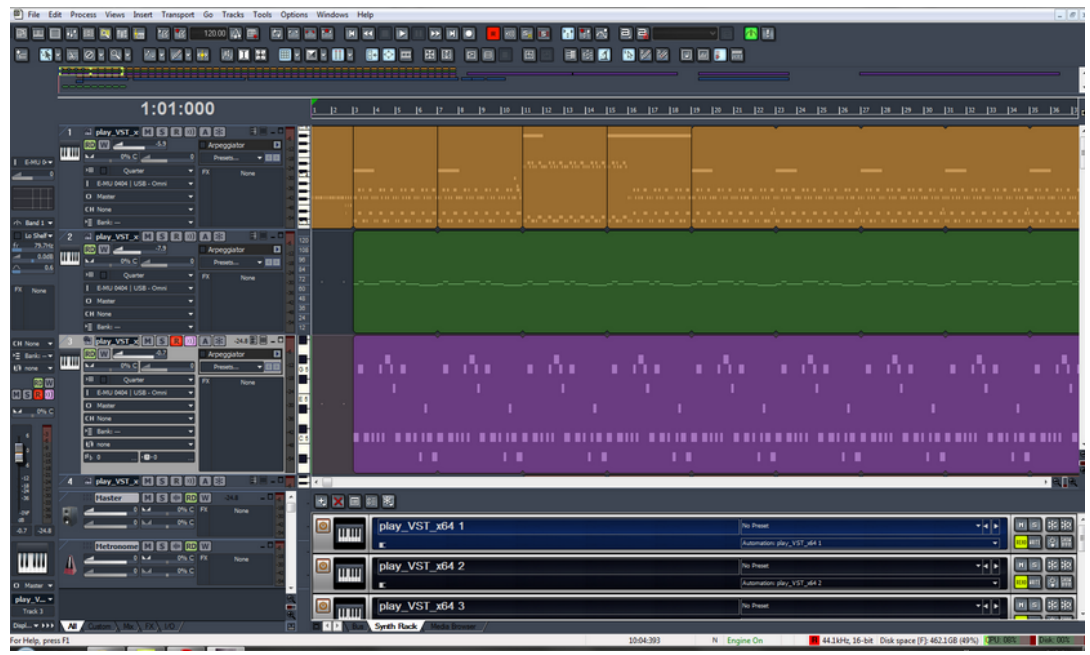

Figure 1. The Computer Music Composition Platform

\section{The Proposed Perspective}

The Features of the Big Data. Data mining as a new multidisciplinary cross application field is the decision support from all walks of life activities plays an increasingly important role. With the rapid development of information technology, all walks of life have accumulated the massive amounts of the heterogeneous data. The task of data mining is the use of various analysis tools found in the huge amounts of data model and the relationships between data and in practice, it can be divided into: classification, clustering, discovery of association rules and general sequential patterns found, outlier analysis, etc. The process of the data mining can be roughly divided into: problem definition, the data collection and pretreatment, data mining algorithm, and interpretation of the results and evaluation while the visualization procedure plays an important role in every stage. 


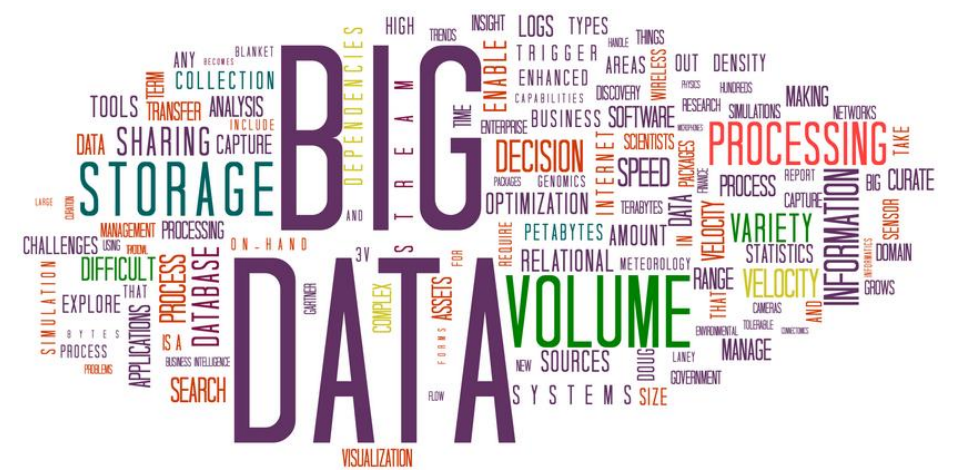

Figure 2. The Keywords of the Big Data

Huge amounts of general data and knowledge poverty leads to the emergence of data mining and knowledge discovery research as knowledge discovery from data to identify valid, novel, potentially useful and ultimately understandable patterns of the nontrivial process. Now the knowledge discovery basically has two branches, respectively database knowledge discovery and the knowledge discovery based on literature. Data mining is divided into three stages: data preparation, data mining, the results of evaluation and expression. Data preparation is mainly to complete the selection of a large amount of data, purification, conjecture, conversion, data reduction, data preparation work is good or bad will affect the efficiency and accuracy of data mining and the effectiveness of the final model, the data preparation phase can eliminate useless data in the process of mining, so as to improve the efficiency and the accuracy of the data mining; Data mining work first need to select the corresponding mining algorithm implementation, such as decision trees, classification, clustering and rough set, association rule, neural network and genetic algorithm, and then analyze the data, thus obtains knowledge model; The results of evaluation and expression, is to determine the pattern of knowledge model is effective to find meaningful. Correspondingly, the related procedures could be summarized as the follows.

- Model representation. It is the language used to describe to find models. If the description of the language ability is stronger, will help to find the precise mathematical model. Ability more description language, however, it is likely to lead to the found model too general, to reduce the accuracy of the forecast.

- Discovery method. Divided into model parameter detection and the discovery. After model representation and model evaluation standard is determined, the data mining was completely turned into the optimization task, that is, from the description of the data found in the most suitable parameter evaluation standard or the model. Specifically the parameter is found after the determination data sets to find the most suitable for model evaluation standard parameter.

- The prediction model of a class can make use of some test data set to evaluate its precision. To describe the model of the class, can be in accuracy, novelty, the practicability and evaluate the various aspects such as understandability [7].

The Digital Music. The development of modern information technology, more and more of the printed material into the library, tapes, video tapes, CD, VCD, DVD, LD DVD and other audio-visual materials account for a large part of this part of the data we referred to as the book. Compared with books and periodicals, audio-visual materials can be fully arouse readers' visual and auditory organs, to give readers the real vivid sensory stimulation, deepen information acquisition and therefore very 
popular with readers. Electronic music in the music philosophy broke through the traditional mode of thinking, from the content of the music to the form of music, from the creation of the music form the communication method of the music, from the organizational principle of the music to the music the development of logic. Concepts such as traditional music creation organization notes in the form of linear thinking, electronic music is based on nonlinear thinking way of organizing music, namely, of the original sound recording, sampling, etc., the middle of the voice processing, bureau of rail and the local collage, all kinds of montage editing and the local sound synthesis, as well as the overall music synthesis of late. Traditional music is formed in a relatively stable environment music system and the system size and sound of music, even if there is a variety of creative ways to explore, deconstruction music system, but only in the aspect of music composition is more complicated. But electronic music radically deconstructs the sound system, blurs the absolute limit, music and noise directly to all kinds of sound into music has realized the sound source of infinite.

In the sound production is done by a computer program to set up and, in the voice processing and program to set up and completed by computer, the computer music whether it is generated by means of computer language programming, organization and control, or in the computer algorithm for main technical way to handling and processing, are based on the computer as the platform, as a result of the electronic equipment and information technology innovation and basic development tend to be more computerized, informatization and networking, the rapid development of it has become the trend.

The Data Based Computer Music Composition. Using the computer music would not have these differences first of all the computers can make easier coding of the composer as can real record composer's information in detail. At the same time, the computer also reduces degree of difficulty of decoding the singer and musician, the transformation of this process is accomplished by a computer. Composer only need to complete the regular playing on the keys, these performance information can automatic coding on the computer, which is the true content of the digital coding.

Type of music, each has its own characteristic of the basic tune. For example, instrumental in the creation, the creation of the tune to qualify for certain musical instrument or voice quality range, the sound of playing techniques, different tone and the effect after combined with other instruments, etc. Therefore, emphasize the traditional tune creation and combining the particularity of computer music melody composing practice constitute and many possibilities, to master different characteristic music melody composed of technical skills and methods, to create with the characteristics of age, living and moving music. In the figure three, we show data based computer music composition characteristics. 


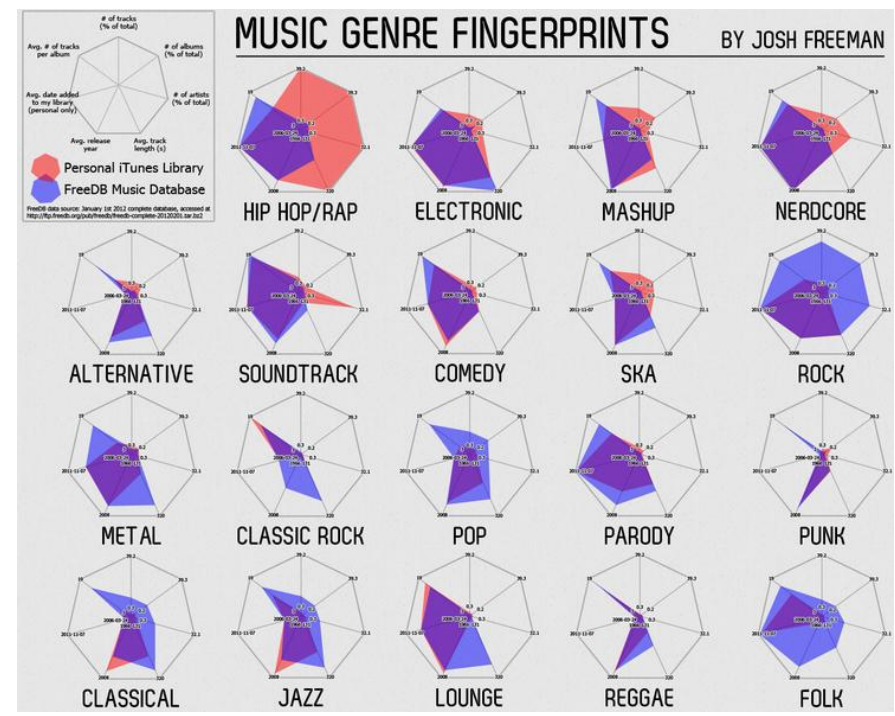

Figure 3. The Data Based Computer Music Composition Characteristics

Computer to songs is broke the pattern, can imitate the conventional instrument timbre, the use of the music of the material as the inspiration of creation, creation and invention of timbre. Composer can voice of acquisition work in nature can be the animal chirping and roar, also can be the wind, the sound of water, sound waves, and even industrial noise, etc., these are rich in content.

Shrinks mixes in the late in the applications of computer music, recording technology is very important, is an important link in the late audio processing, its purpose is to make the late products get better sound effect. Mainly do early music compression, make the music sounds more balanced, in mastering the principle of overall balance to late shrinks mixes music works until finish making the music. Master mixing link, the composer's understanding of timbre is deeper, mixing, in the late, as it were and is directly related to the quality of the music works and the sound effect. Use the MIDI sequencer within a variety of convenient way to input and editing and typesetting function, and can quickly input and edit music info, and will be music, audio output at the same time. In this way, the computer becomes a convenient and practical stereo synthesizer and the score of the processor. Audio editing in the MIDI music program has become more and more powerful to make electronic music elements can be added directly in the MIDI music, namely digital waveform recording, the editing functions can use MIDI sequencer internal waveform processing program, or can synchronize with MIDI sequencer waveform sequence program for prefabricated and deformation. As a result, these methods and techniques, that can guide the composer of music creation, is the core part of music creation. Computer composition is a traditional method of composition and music software actual operation with the combination of a creative way, to learn the operation of the music software are relatively difficult, but for music creation, traditional composing method of general learning is more important and in practice of the computer music, traditional music composition method is integration.

\section{Conclusion}

In this paper, we conduct research on the accurate computer music composition mode under the basic background of big data. In the song creation, computer music creation is an important component, and occupies more important position as the core creation of the computer has broken the original model, improves the plasticity of music and limitlessness, let music creation more flexible as is an important way of 
creation. Computer technology has changed the music not only, also changed the way creation, enrich the forms of computer music creation, to improve the scope of creation. The computer music creation to break through the existing music style has obvious advantages this also is a focus in the study of computer creation. Our research reviews the state-of-the-art approaches of making the digital music that serves as the novel paradigm for further development.

\section{References}

[1] Colwell, Cynthia M., et al. "Impact of music therapy interventions (listening, composition, Orff-based) on the physiological and psychosocial behaviors of hospitalized children: a feasibility study." Journal of pediatric nursing 28.3 (2013): 249-257.

[2] Biasutti, Michele. "Assessing a collaborative online environment for music composition." Journal of Educational Technology \& Society 18.3 (2015): 49-63.

[3] Hogenes, M., et al. "Effects of a Music Composition Intervention on Elementary School Children." (2013).
[4] Kim, Keunhyoung Luke, and Woon Seung Yeo. Griddy: a Drawing Based Music Composition System with Multi-layered Structure. Ann Arbor, MI: Michigan Publishing, University of Michigan Library, 2014.

[5] Richmond, James, et al. "Engagement and skill development through an innovative classroom music program." International Journal of Music Education (2015): 0255761415584289.

[6] Hopkins, Michael T. "Collaborative Composing in High School String Chamber Music Ensembles." Journal of Research in Music Education 62.4 (2015): 405-424.

[7] Huang, Chih-Fang, and En-Ju Lin. "Using Emotion Map System to Implement the Generative Chinese Style Music with $\mathrm{Wu}$ Xing Theory." Advanced Research and Trends in New Technologies, Software, Human-Computer Interaction, and Communicability (2013): 183. 